\title{
Boron deactivation in preamorphized silicon on insulator: Efficiency of the buried oxide as an interstitial sink
}

\author{
J. J. Hamilton ${ }^{\text {a) }}$ and K. J. Kirkby \\ Ion Beam Centre, Advanced Technology Institute, University of Surrey, Guildford, Surrey GU2 7XH, \\ United Kingdom \\ N. E. B. Cowern \\ Institute for Nanoscale Science and Technology and School of Electrical, Electronic and Computer \\ Engineering, University of Newcastle upon Tyne, Newcastle upon Tyne NE1 7RU, United Kingdom \\ E. J. H. Collart \\ Parametric and Conductive Implant Division, Applied Materials UK Ltd., Foundry Lane, Horsham, \\ West Sussex RH13 5PX, United Kingdom \\ M. Bersani, D. Giubertoni, and S. Gennaro \\ ITC-irst Centro per la Ricerca Scientifica e Tecnologica, ITC-irst, 38050-Povo Trento, Italy
}

\begin{abstract}
A. Parisini
CNR-IMM Sezione di Bologna, Via Gobetti 101, 40129 Bologna, Italy
\end{abstract}

(Received 13 November 2006; accepted 10 August 2007; published online 30 August 2007)

\begin{abstract}
Preamorphization of ultrashallow implanted boron in silicon on insulator is optimized to produce an abrupt boxlike doping profile with negligible electrical deactivation and significantly reduced transient enhanced diffusion. The effect is achieved by positioning the as-implanted amorphous/ crystalline interface close to the buried oxide interface to minimize interstitials while leaving a single-crystal seed to support solid-phase epitaxy. Results support the idea that the interface between the Si overlayer and the buried oxide is an efficient interstitial sink. (C) 2007 American Institute of Physics. [DOI: 10.1063/1.2778749]
\end{abstract}

Highly activated ultrashallow boxlike doping profiles are an important requirement for advanced planar complementary metal oxide semiconductor (MOS) applications. ${ }^{1}$ A promising fabrication approach for $p$-channel MOS transistors is the use of Ge preamorphizing implants (PAIs) prior to ultralow energy B implantation. This reduces channeling and increases electrical activation due to solid-phase epitaxial regrowth (SPER). ${ }^{2}$ For future technology nodes, bulk Si wafers may be replaced by silicon on insulator (SOI). ${ }^{3}$

Unfortunately, in both bulk Si and SOI, the PAI implantation step causes an interstitial-rich "end-of-range" (EOR) defect band to form on further annealing. As this EOR band evolves, it releases self-interstitials, causing transient enhanced diffusion (TED) and boron-interstitial cluster (BIC) formation which manifests itself as electrical deactivation. ${ }^{4}$ In SOI, a proportion of the emitted self-interstitials may migrate to nearby sinks such as the silicon/buried oxide (BOX) interface, thus reducing the amount of $\mathrm{B}$ deactivation that occurs. 5

This letter quantifies the role of the BOX and shows the dramatic advantages - in terms of reduced diffusion and deactivation-that can be achieved by exploiting the positioning of the EOR defect band within the silicon top layer in SOI. We will show that two distinct physical processes are involved: the removal of interstitials at the BOX interface following diffusion within the Si overlayer and the cutting off of the "as-implanted" excess interstitial profile within the BOX.

Experiments were performed on $n$-type $\langle 100\rangle$ Czochralski $\mathrm{Si}$ wafers with a resistivity of $\sim 10-25 \Omega \mathrm{cm}$, and on

${ }^{a)}$ Electronic mail: j.hamilton@eim.surrey.ac.uk
SOITECC SOI wafers with a nominal $145 \mathrm{~nm}$ BOX and a $55 \mathrm{~nm}$ p-type Si overlayer. The wafers were implanted with $\mathrm{Ge}^{+}$at $8,20,24,28,32$, or $36 \mathrm{keV}$ to a dose of 1 $\times 10^{15} \mathrm{~cm}^{-2}$, amorphizing to depths of $\sim 20,40,45,50,55$, and $60 \mathrm{~nm}$, respectively, as determined by Rutherford backscattering spectrometry (RBS) and cross-sectional transmission electron microscopy (XTEM). Boron was subsequently implanted at $500 \mathrm{eV}$ to a dose of $2 \times 10^{15} \mathrm{~cm}^{-2}$ at $0^{\circ}$ tilt and $0^{\circ}$ twist. RBS used a $1.5 \mathrm{MeV} \mathrm{He}$ beam at $45^{\circ}$ to the sample. $^{6}$

After implantation, samples received isochronal rapid thermal annealings in $\mathrm{N}_{2}$ ambient, using a Process Products Corporation 18-lamp system (with lamps above and below the sample) operating with a set time of $60 \mathrm{~s}$ at temperatures in the range of $700-1000{ }^{\circ} \mathrm{C}$, considered suitable for a study of $\mathrm{B}$ activation. The electrical activation (sheet resistance $R_{S}$ and active dose $N_{s}$ ) of samples was analyzed by Hall measurements using an Accent HL5500 machine. Atomic profiles of B in selected samples were measured by secondary ion mass spectrometry (SIMS) using a CAMECA Wf SCULTRA instrument operating with a $0.5 \mathrm{keV} \mathrm{O}_{2}^{+}$primary beam at $70^{\circ}$ incidence. Oxygen flooding and stage rotation were used to avoid ripple formation. ${ }^{7}$ The presence and structure of extended defects were investigated by XTEM with a weak-beam (WB) setting of $(-\mathbf{g}, \mathbf{g})$ with $\mathbf{g}=\langle 400\rangle$, using a FEI Tecnai F20 electron microscope operating at $200 \mathrm{kV}$.

Amorphous layer regrowth and the quality of the resultant crystalline $\mathrm{Si}$ were monitored by $\mathrm{RBS}^{8,9}$ A comparison of regrowth rates in bulk $\mathrm{Si}$ and SOI samples showed that any systematic difference between the temperatures in the two sample types was less than $3{ }^{\circ} \mathrm{C}$, negligible for the pur- 


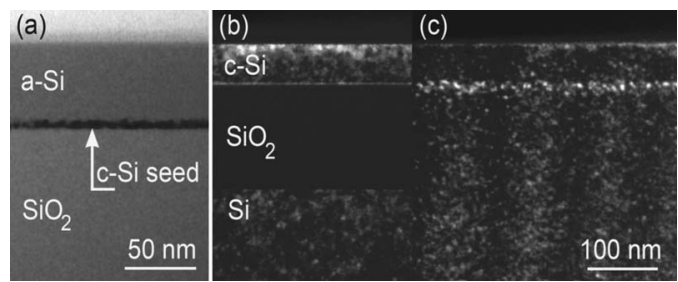

FIG. 1. XTEM micrographs of $32 \mathrm{keV}$ Ge PAI B implanted SOI and bulk Si samples. (a) BF micrograph of an as-implanted SOI sample. [(b) and (c)] WB $(-\mathbf{g}, \mathbf{g}) \mathbf{g}=\langle 400\rangle$, XTEM micrographs of samples annealed at $700{ }^{\circ} \mathrm{C}$ for $60 \mathrm{~s}$ in SOI and bulk Si, respectively.

pose of this study. In all SOI samples amorphized at $32 \mathrm{keV}$ and below, the Si overlayer regrew fully into single-crystal $\langle 100\rangle \mathrm{Si}$. In contrast, SOI samples preamorphized at $36 \mathrm{keV}$ did not regrow into a single crystal.

Figure 1(a) shows a bright-field (BF) XTEM micrograph for the case of as-implanted $32 \mathrm{keV}$ Ge in SOI. A thin $(<4 \mathrm{~nm})$ layer of single-crystal $\mathrm{Si}$ remains between the amorphous layer and the upper BOX interface. Figures 1(b) and 1(c) show WB dark field micrographs for SOI and bulk Si samples with the same implant condition, after annealing at $700{ }^{\circ} \mathrm{C}$ for $60 \mathrm{~s}$. Here, the amorphous layer is no longer visible and the regrown region consists of single-crystal Si. In the bulk case [Fig. 1(c)], a band of EOR defects is visible at a depth of $\sim 60 \mathrm{~nm}$ below the sample surface, but in the SOI [Fig. 1(b)] no significant defect accumulation is found, and high-resolution imaging (not shown) detects only a small number of isolated $\{113\}$ defects close to the BOX interface. A plausible explanation for this effect is that part of the reservoir of excess interstitials that nucleate into EOR defects in the bulk case is absent in the SOI case. The excess atom density generated by the Ge implant in the SOI BOX remains trapped there, as the diffusivity of $\mathrm{Si}$ in $\mathrm{SiO}_{2}$ is negligible at the temperatures used in this study. ${ }^{10}$ Only the excess interstitials created in the thin crystalline region between the amorphous/crystalline $(a / c)$ interface and the BOX interface are available to nucleate into EOR defects, and of these an unknown proportion may be consumed in interface reactions.

Figure 2 reports the electrical response of SOI samples with PAI conditions in the range of $8-32 \mathrm{keV}$. At low PAI energies, the characteristic deactivation and reactivation of $\mathrm{B}$

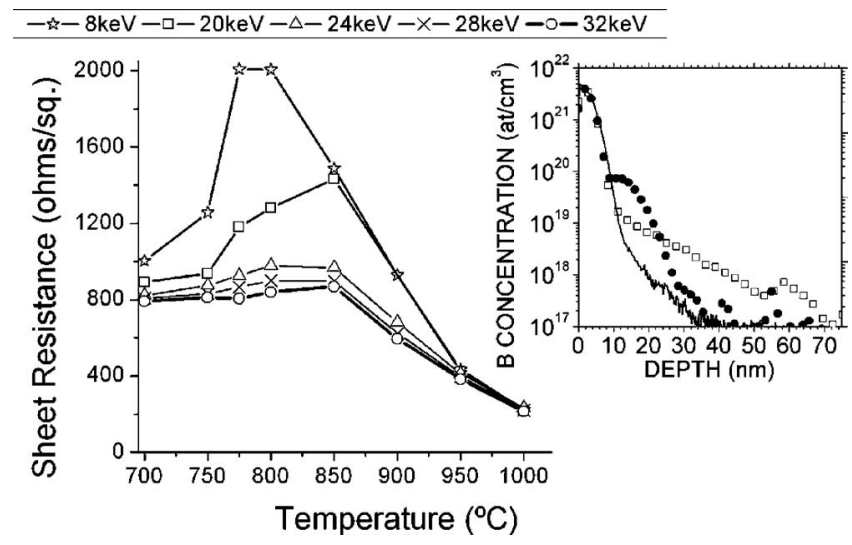

FIG. 2. van der Pauw resistivity after $60 \mathrm{~s}$ isochronal annealings. Open stars, squares, triangles, crosses, and circles represent 8, 20, 24, 28, and $32 \mathrm{keV}$ Ge PAI in SOI, respectively. Inset are SIMS profiles of as implanted (solid line), $32 \mathrm{keV}$ Ge PAI in bulk $\mathrm{Si}$ (open squares), and SOI (closed

circles) annealed at $850{ }^{\circ} \mathrm{C}$ for $60 \mathrm{~s}$ carriers at $700{ }^{\circ} \mathrm{C}$ and that at the maximum measured deac-
Downloaded 30 Mar 2009 to 131.227 .178 .132$. Redistribution subject to AlP license or copyright; see http://apl.aip.org/apl/copyright.jsp

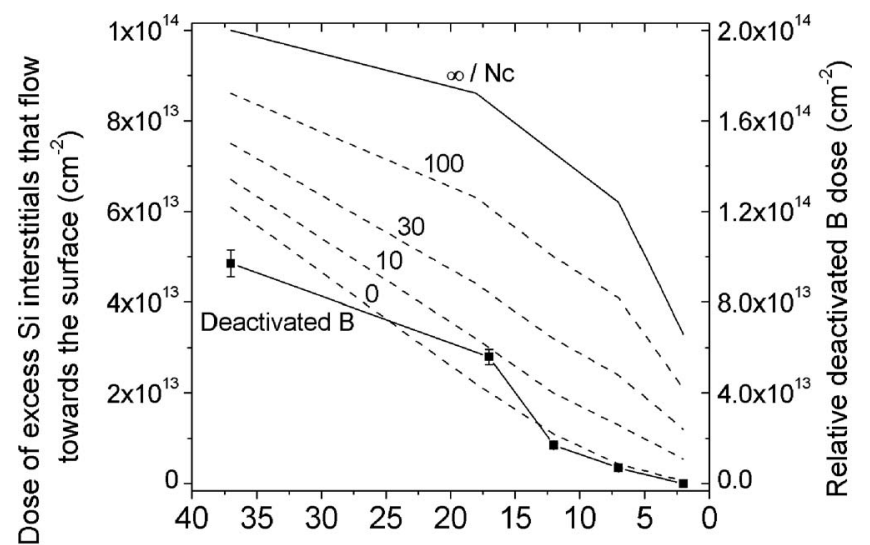

Remaining crystal thickness after amorphization $(\mathrm{nm})$

FIG. 3. Dose of excess Si interstitials that flow toward the surface causing deactivation for various values of recombination length $\left(L_{2}\right.$ $=0,10,30,100, \infty)$, plotted together with the deactivated $\mathrm{B}$ dose. The results are plotted as a function of the remaining crystal layer thickness after amorphization. The above quantities are represented by lines and symbols, respectively (error bars in some cases are smaller than symbol size).

are observed, arising from the formation and subsequent dissolution of BICs. ${ }^{4}$ However, as the PAI energy is increased, bringing the EOR defect band toward the BOX interface, the amount of deactivation decreases very dramatically.

The effects in terms of diffusion can be seen from SIMS profiles for the $32 \mathrm{keV}$ PAI case, after annealing at $850{ }^{\circ} \mathrm{C}$, shown in the inset to Fig. 2. Comparing SOI to bulk Si, the amount of TED in the SOI is dramatically reduced, the "kink" concentration where the B diffuses out from the implant is higher indicating better activation, and the junction is much more abrupt. Indeed, the kink level in the SOI is above the equilibrium solubility limit for B in crystalline silicon-a result which is unprecedented in material that has not been coimplanted to suppress TED. These beneficial effects indicate that the number of interstitials reaching the B-doped near surface region has been greatly reduced-again consistent with our conclusion from the TEM analysis.

At this point, the question arises as to whether, contrary to previously published arguments concerning interface recombination, ${ }^{11}$ all of our results might be explained in terms of the locking of the excess interstitial distribution within the BOX. To test this hypothesis, we estimate the dose of excess interstitials implanted in the residual crystalline portion of the Si overlayer as a function of PAI energy, using the kinetic Monte Carlo implant simulator, KING. ${ }^{12}$

The excess interstitial distributions for the as-implanted SOI samples were integrated between the amorphous/ crystalline interface and the BOX interface to give the dose of excess interstitials remaining within the overlayer after amorphization. The same calculation for bulk silicon, where the $\mathrm{BOX}$ is absent, gave a correspondingly larger dose of excess interstitials. Comparison of this calculated dose to experimental measurements of the number of interstitials in the EOR band in bulk silicon ${ }^{13}$ then allowed us to normalize the calculations for SOI to give the number of excess interstitials $N_{C}$ in the EOR band for each Ge implant energy in SOI. The resulting value of $N_{C}$ is shown in Fig. 3 as a continuous line. In the same plot, we show the amount of deactivation caused by the interstitials from the EOR band, expressed as the difference, $\delta N_{B}(E)$, between the number of carriers at $700{ }^{\circ} \mathrm{C}$ and that at the maximum measured deac- 
tivation (corresponding to the $R_{s}$ peaks in Fig. 2) for each PAI energy. The assumptions made here are as follows: (i) no significant BIC dissolution occurs simultaneously to the EOR dissolution, (ii) during the deactivation phase a high percentage (approximated as 100\%) of the interstitials in the EOR defects are released. The data are presented in the form $\delta N_{B}(E)-\delta N_{B}(32 \mathrm{keV})$ to eliminate the effect of the small amount of "equilibrium" deactivation that occurs in the $32 \mathrm{keV}$ case as a result of relaxation of the dopant from its initially high solubility in amorphous silicon toward its solubility in crystalline silicon. This effect is unrelated to the presence of excess interstitials, occurring even in silicon that has been treated with an excess of vacancies. ${ }^{14}$

Figure 3 shows that, as the thickness of the remaining crystalline layer is reduced, B deactivation decreases much more rapidly than the number of available interstitials. A qualitative explanation is that, as the EOR band approaches the interface with the BOX, an increasing proportion of interstitials is absorbed there instead of migrating to the near surface region. In order to quantify this picture, we use the following simplified model. The EOR band is treated as a simple "plane" at depth $x$, placed at the centroid of the excess interstitial depth distribution in the region beyond the $a / c$ interface. This approximation enables a simple calculation of the interstitial fluxes toward the top and bottom interfaces of the $\mathrm{Si}$ overlayer during annealing, $\varphi_{1}=D_{I} C_{I, \mathrm{EOR}} /(x$ $\left.+L_{1}\right), \varphi_{2}=D_{I} C_{I, \mathrm{EOR}} /\left(d-x+L_{2}\right)$, where $D_{I}$ is the interstitial diffusivity, $C_{I, \mathrm{EOR}}$ is the interstitial concentration at the EOR depth, $d$ is the thickness of the $\mathrm{Si}$ overlayer, and $L_{1}$ and $L_{2}$ are the recombination lengths for interstitials at its upper and lower interfaces, respectively.

The fraction of interstitials flowing to the surface is then given by $F_{1}=\left(d-x+L_{2}\right) /\left(d+L_{1}+L_{2}\right)$. As is well known, ${ }^{15}$ the recombination length at the surface, $L_{1}$, is less than a few nanometers during TED and can certainly be neglected in comparison to the thickness $d$. We therefore have as a good approximation $F_{1}=\left(d-x+L_{2}\right) /\left(d+L_{2}\right)$. This quantity, multiplied by the dose $N_{C}$, is plotted as a family of curves in Fig. 3 for the cases, $L_{2}=(0,10,30,100, \infty) \mathrm{nm}$. In the case $L_{2}$ $\rightarrow \infty$, for which $F_{1} \rightarrow 1$, the BOX interface is modeled as a reflecting boundary for interstitials, whereas in the case $L_{2}$ $=0$, it is a perfect sink.

By treating the number of $\mathrm{B}$ atoms deactivated by one $\mathrm{Si}$ interstitial, $n$, as a free parameter in a fit of $F_{1} N_{C}$ to the deactivation data, we find the best fit for the case $L_{2}=0$, and a significant increase in $\chi^{2}$ for the $L_{2}>10$. Moreover, the fitted value of $n$ is $\sim 2$ for the case $L_{2}=0$, but becomes unrealistically small $(n<1)$ for larger $L_{2}$ values. In Fig. 3, we have chosen to compare the number of interstitials reaching the surface with the measured deactivation, using vertical scales differing by a factor of 2 , corresponding to the usually observed deactivation of about two $\mathrm{B}$ atoms by one interstitial. ${ }^{16,17}$ The agreement is good for all data points except the first (remaining crystal thickness of $37 \mathrm{~nm}$ ) for which the EOR band slightly overlapped the B implant distribution. The slightly low value for this point could be a result of direct deactivation by implanted interstitials prior to annealing. The shape of the actual deactivation curve is clearly best matched by a value of $L_{2}=0$, indicating that the $\mathrm{BOX}$ interface is an efficient sink and plays an important role in the control of defects, deactivation, and TED in SOI layers. Recent quantitative TEM data ${ }^{18}$ show a similar trend, with a strong dependence on distance between EOR and BOX.

In conclusion, we have demonstrated and explained an optimal approach for preamorphization in SOI. Two phenomena are involved: (a) the SOI BOX interface acts as an efficient sink for self interstitials and (b) the overlap of the interstitial profile with the BOX interface cuts the initial number of interstitials within the Si overlayer. The consequent reduction in the number of interstitials driving BIC formation in the near surface region leads to more stable electrical activation, less TED, and a more abrupt junction. The optimum result is achieved by tailoring the Ge PAI to ensure that the amorphous/crystalline interface is placed as close as possible to the $\mathrm{BOX}$, consistent with the requirement to maintain a single-crystal $\mathrm{Si}$ layer adjacent to the upper BOX interface for reliable SPER.

The authors thank Roger Webb, Andrew Smith, Norman Kirkby, and Giorgio Lulli for useful input and fruitful discussions on the simulations. Part of this work was supported by the U.K. Engineering and Physical Sciences Research Council (EPSRC) and the Surrey Ion Beam Centre.

${ }^{1}$ The International Technology Roadmap for Semiconductors (2006).

${ }^{2}$ R. Lindsay, B. Pawlak, J. Kittl, K. Henson, C. Torregiani, S. Ciangrandi, R. Surdeanu, W. Vandervorst, A. Mayur, J. Ross, S. McCoy, J. Gelpey, K. Elliott, X. Pages, A. Satta, A. Lauwers, P. Stolk, and K. Maex, Mater. Res. Soc. Symp. Proc. 765, D7.4.1 (2003).

${ }^{3}$ C. K. Celler and S. Cristoloveanu, J. Appl. Phys. 93, 4955 (2003).

${ }^{4}$ B. Colombeau, A. J. Smith, N. E. B. Cowern, B. J. Pawlak, F. Cristiano, R. Duffy, A. Claverie, C. J. Ortiz, P. Pichler, E. Lampin, and C. Zechner, Mater. Res. Soc. Symp. Proc. 810, C3.6.1 (2004).

${ }^{5}$ J. J. Hamilton, N. E. B. Cowern, E. J. H. Collart, B. Colombeau, M. Bersani, D. Giubertoni, A. Parisini, J. A. Sharp, and K. J. Kirkby, Appl. Phys. Lett. 89, 042111 (2006).

${ }^{6}$ C. Jeynes, N. P. Barradas, P. K. Marriot, G. Boudreault, M. Jenkin, E. Wendler, and R. P. Webb, J. Phys. D 36, R97 (2003).

${ }^{7}$ M. Bersani, D. Giubertoni, E. Iacob, M. Barozzi, S. Pederzoli, L. Vanzetti, and M. Anderle, Appl. Surf. Sci. 252, 7315 (2006).

${ }^{8}$ J. J. Hamilton, E. J. H. Collart, B. Colombeau, C. Jeynes, M. Bersani, D. Giubertoni, J. A. Sharp, N. E. B. Cowern, and K. J. Kirkby, Nucl. Instrum. Methods Phys. Res. B 237, 107 (2005).

${ }^{9}$ G. L. Olson, Mater. Res. Soc. Symp. Proc. 35, 25 (1985).

${ }^{10}$ D. Tsoukalas, C. Tsamis, and P. Normand, Mater. Res. Soc. Symp. Proc. 669, J3.7 (2001).

${ }^{11}$ A. F. Saavedra, A. C. King, K. S. Jones, E. C. Jones, and K. K. Chan, J. Vac. Sci. Technol. B 20, 2243 (2002).

${ }^{12}$ G. Lulli, E. Albertazzi, M. Bianconi, R. Nipoti, M. Cervera, A. Carnera, and C. Cellini, J. Appl. Phys. 82, 5958 (1997).

${ }^{13}$ A. Claverie, B. Colombeau, B. Mauduit, C. Bonafos, X. Herbras, G. Assayag, and F. Cristiano, Appl. Phys. A: Mater. Sci. Process. 76, 1025 (2003).

${ }^{14}$ A. J. Smith, N. E. B. Cowern, B. Colombeau, R. Gwilliam, B. J. Sealy, E. J. H. Collart, S. Gennaro, D. Giubertoni, M. Bersani, and M. Barozzi, AIP Conf. Proc. 866, 84 (2006).

${ }^{15}$ N. E. B. Cowern, D. Alquier, M. Omri, A. Claverie, and A. Nejim, Nucl. Instrum. Methods Phys. Res. B 148, 257 (1999).

${ }^{16}$ B. Colombeau, A. J. Smith, N. E. B. Cowern, B. J. Pawlak, F. Cristiano, R. Duffy, A. Claverie, C. J. Ortiz, P. Pichler, E. Lampin, and C. Zechner, Mater. Res. Soc. Symp. Proc. 810, C3.6.1 (2004).

${ }^{17}$ B. J. Pawlak, R. Surdeanu, B. Colombeau, A. J. Smith, N. E. B. Cowern, R. Lindsay, W. Vandervorst, B. Brijs, O. Richard, and F. Cristiano, Appl. Phys. Lett. 84, 2055 (2004).

${ }^{18}$ P. F. Fazzini, F. Crisitano, C. Dupré, A. Claverie, T. Ernst, and M. Gavelle, J. Vac. Sci. Technol. B (in press). 